Boise State University

ScholarWorks

8-1-2013

\title{
A Framework for Understanding Noise Impacts on Wildlife: An Urgent Conservation Priority
}

Clinton D. Francis

National Evolutionary Synthesis Center

Jesse R. Barber

Boise State University 


\title{
A framework for understanding noise impacts on wildlife: an urgent conservation priority
}

\author{
Clinton D Francis ${ }^{1 * \dagger}$ and Jesse R Barber ${ }^{2 \dagger}$
}

Anthropogenic noise is an important environmental stressor that is rapidly gaining attention among biologists, resource managers, and policy makers. Here we review a substantial literature detailing the impacts of noise on wildlife and provide a conceptual framework to guide future research. We discuss how several likely impacts of noise exposure have yet to be rigorously studied and outline how behavioral responses to noise are linked to the nature of the noise stimulus. Chronic and frequent noise interferes with animals' abilities to detect important sounds, whereas intermittent and unpredictable noise is often perceived as a threat. Importantly, these effects can lead to fitness costs, either directly or indirectly. Future research should consider the range of behavioral and physiological responses to this burgeoning pollutant and pair measured responses with metrics that appropriately characterize noise stimuli. This will provide a greater understanding of the mechanisms that govern wildlife responses to noise and help in identifying practical noise limits to inform policy and regulation.

Front Ecol Environ 2013; 11(6): 305-313, doi:10.1890/120183 (published online 15 Jul 2013)

$\mathrm{A}^{\mathrm{n}}$ emerging aim in applied ecology and conservation biology is to understand how human-generated noise affects taxonomically diverse organisms in both marine (eg Slabbekoorn et al. 2010; Ellison et al. 2012) and terrestrial (eg Patricelli and Blickley 2006; Barber et al. 2010; Kight and Swaddle 2011) environments. Noise is a spatially extensive pollutant and there is growing evidence to suggest that it may have highly detrimental impacts on natural communities; yet efforts to address this issue of emerging conservation concern lack a common framework for understanding the ecological consequences of noise. A conceptual scaffold is critical to scientific progress and to

\section{In a nutshell:}

- Noise is an intense, widespread pollutant, relevant to conservation efforts worldwide

- Using the number of animals present in environments exposed to anthropogenic noise as the sole metric of noise impacts can be deceiving because there are many hidden costs of noise exposure (eg compromising predator/prey detection or mating signals, altering temporal or movement patterns, increasing physiological stress)

- To ensure that conservation initiatives (and efforts to establish regulatory limits) are relevant, investigators must properly characterize a suite of noise features

- Reducing noise exposure and incorporating sound measurement into environmental planning will quickly benefit ecological systems

${ }^{1}$ National Evolutionary Synthesis Center, Durham, NC; current address: Biological Sciences Department, California Polytechnic State University, San Luis Obispo, CA *(cdfranci@calpoly.edu); ${ }^{2}$ Department of Biological Sciences, Boise State University, Boise, ID; the authors contributed equally to this work its ability to inform conservation policy. As more attention and resources are invested in understanding the full ecological effects of noise, it is important that investigators design research questions and protocols in light of the many possible costs associated with noise exposure and also that they properly link responses to several relevant features of noise, such as intensity, frequency, or timing, that could explain wildlife responses (Panel 1).

Here we introduce a framework using a mechanistic approach for how noise exposure can impact fitness at the level of the individual organism as a result of changes in behavior, and identify several acoustic characteristics that are relevant to noise exposure and ecological integrity. We provide representative examples of noise impacts, primarily from terrestrial systems; however, these issues are equally applicable to organisms in aquatic environments. We stress that various responses to noise exposure are less obvious than those that have typically been studied to date, such as signal modifications (eg changes in vocal frequency, amplitude, or vocalization timing) and decreases in site occupancy (eg Bayne et al. 2008; Francis et al. 2011b). Importantly, probable behavioral responses to noise that merit further scientific study might be detrimental to individual fitness and may have severe populationlevel consequences. As we show below, the presence of a species in a noisy area cannot be interpreted as an indication that it is not being impacted by elevated sound levels, because there are many potential costs associated with noise exposure that have not been rigorously studied.

Variation in responses to the same noise stimulus

Species differ in their sensitivities to noise exposure (Bayne et al. 2008; Francis et al. 2009, 2011a); however, 


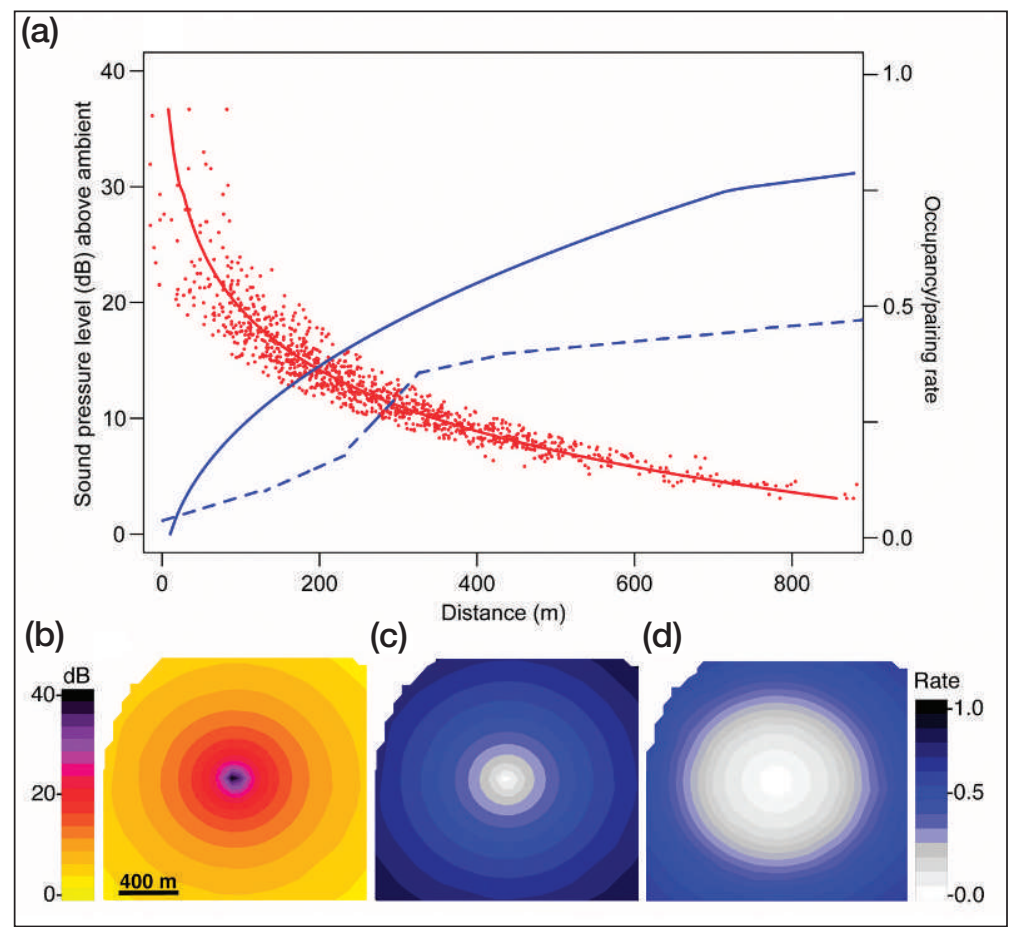

Figure 1. Responses to the same noise stimulus can take a variety of shapes. (a) The sound pressure level (SPL) of noise (red) decreases with increasing distance from the source but may not reach "baseline" ambient levels until $\sim 1 \mathrm{~km}$ away (this distance will vary depending on noise source and the environment). Response curves for species occupancy (blue solid line) and pairing rates (blue dashed line) in response to noise may have unique shapes, as might other measures of species responses to noise stimuli. The relationship between SPL and distance is from Francis et al. (2011c) and Francis (unpublished data) with noise generated from gas well compressors. Behavioral responses are hypothetical but based on responses in Francis et al. (2011c). (b) Spatial propagation of elevated noise levels from a point source (such as a single car or an oil/gas compressor station), which decays at a spreading loss of $6 \mathrm{~dB}$ or more per doubling of distance, due to the geometry of the spherical wave front. It is important to note that line sources (such as a busy highway; not shown) lose only $3 \mathrm{~dB}$ per doubling of distance due to their cylindrical wave front. Clearly, knowledge of the geometry of anthropogenic noise stimuli is essential to understanding the scale of exposure. ( $c$ and d) Spatial representation of (c) species occupancy and (d) pairing success surrounding a point source of noise.

the degree to which individuals vary in sensitivity to noise during each life-history stage or due to behavioral context has been underappreciated. For example, ovenbird (Seiurus aurocapilla) habitat occupancy appears uninfluenced by noise exposure (Habib et al. 2007; Bayne et al. 2008; Goodwin and Shriver 2011), yet males defending noisy territories are less successful in attracting mates (Habib et al. 2007). Reed buntings (Emberiza schoeniclus) also show reduced pairing success in noisy areas (Gross et al. 2010). Such examples should serve as a warning to biologists, land managers, and policy makers: the same noise stimulus can affect various response metrics in different ways. An organism might show little to no response to noise in terms of habitat occupancy or foraging rate, for example, but may experience strong negative impacts in terms of pairing success, number of offspring, physiological stress, or other measures of fitness (Figure 1). Because the various responses may range from linear to threshold functions of noise exposure, investigators should take an integrative approach that incorporates several different metrics (eg density, pairing success, number of offspring), rather than using a single metric to describe how noise influences their study organism. But which alterations in behavior are most likely to occur and which are the most detrimental? These are important questions because funding and logistical constraints ensure that measuring all of the potential impacts of noise is impossible. Fortunately, the nature of sound stimuli can guide investigators toward likely behavioral changes that may influence fitness.

\section{Characterizing noise and the disturbance-interference continuum}

Determining whether a particular noise stimulus is within an organism's sensory capabilities is foremost in importance; if a sound consists of frequencies that are outside of an organism's hearing range, it will not have a direct effect (Panel 1; Figure 2). Provided that an organism can hear the noise stimulus, its acoustic energy could cause permanent or temporary hearing loss, but this might only occur when the animal is extremely close to the source of the noise (Dooling and Popper 2007).

Instead, sounds may have their greatest influence on behavior, which then translates into fitness costs, but how and why noise elicits a response can vary greatly (Figures 2 and 3 ). At one extreme, noise stimuli that startle animals are perceived as threats and generate self-preservation responses (eg fleeing, hiding), which are similar to responses to real predation risk or nonlethal human disturbance (ie the risk-disturbance hypothesis, which posits that animal responses to human activities are analogous to their responses to real predation risk; Frid and Dill 2002). Noise stimuli at this end of the continuum are often infrequent, but are abrupt and unpredictable. At the other end of the continuum, noise can impair sensory capabilities by masking biologically relevant sounds used for communication, detection of threats or prey, and spatial navigation. These noise stimuli tend to be frequent or chronic and their spectral (ie frequency) content overlaps with biologically relevant sounds. Increases in noise intensity (loudness or amplitude) will increase the severity of the impacts, regardless of whether it is perceived as a threat or masks biologically relevant sounds. An important supplement to this dichotomy is 
that limited stimulus processing capacity could be responsible for some detrimental effects. Noise stimuli of various kinds might act as a distraction, drawing the animal's attention to a sound source and thereby impairing its ability to process information perceived through other sensory modalities (Chan et al. 2010). Alternatively, noise may reduce auditory awareness, trigger increased visual surveillance, and compromise visually mediated tasks. The mechanistic details and ecological importance of such distractions still need to be fully explored. Regardless, the conservation implications of understanding the importance of noise as a distractor are not trivial; if distraction is a fundamental route for noise impacts, our concern might spread beyond those frequencies that overlap with biologically relevant signals.

\section{Behavioral changes}

Although a limited number of laboratory studies have suggested that noise may affect gene expression, physiological stress, and immune function directly (Figure 3a; Kight and Swaddle 2011), most noise-related impacts appear to involve behavioral responses across four categories: (1) changes in temporal patterns, (2) alterations in spatial distributions or movements, (3) decreases in foraging or provisioning efficiency coupled with increased vigilance and anti-predator behavior, and (4) changes in mate attraction and territorial defense (Figure 3). As demonstrated below, these disturbance-, distraction-, and masking-mediated behavioral changes could directly impact individual survival and fitness or lead to physiological stress that may then compromise fitness.

\section{Changes in temporal patterns}

Sound stimuli that are perceived as threats can alter temporal patterns; for example, red foxes (Vulpes vulpes) cross busy roads when traffic rates are lower, suggesting noise cues might be affecting the timing of their movements (Figure 3b; Baker et al. 2007). Similarly, noise from boat traffic disrupts the timing of foraging by West Indian manatees (Trichechus manatus), potentially influencing foraging efficiency and energy budgets (Figure 3m; Miksis-Olds et al. 2007). Noise can also change behavior due to interference with cue detection. European robins (Erithacus rubecula) avoid acoustic interference from urban noise by singing at night, when noise levels are lower than during daylight hours (Figure 3c; Fuller et al. 2007). Although this example may appear to be an

\section{Panel 1. Sound features relevant to noise-impact studies}

In the main text we discuss how the spectral (frequency) composition of noise is related to an organism's hearing range and its ability to detect relevant sounds. For these reasons, it is critical that researchers collect sound-level data with an appropriate frequency-weighting filter. For instance, the " $A$ " filter on many sound-level meters is based on equal loudness contours for human hearing; this filter provides a conservative estimate of bird hearing and is the best readily-available weighting for bird studies (Dooling and Popper 2007). However, whether working with birds or other taxa, it is best to simultaneously record and measure the noise using a "flat" frequency filter, then truncate the resulting spectral output to the most relevant frequency range for each species of interest (see below).

Investigators should also avoid the temptation to characterize a noise stimulus as a single decibel value, whether weighted or not, as other metrics that describe the noise are equally important (Figure 2). Time-averaged values, such as equivalent continuous sound level $\left(L_{e q}\right)$, can be extremely informative to describe sounds that are chronic or frequent; however, these integration times do not properly characterize sounds that occur once, infrequently, or more regularly. Instead, measurements integrated over several hours will mischaracterize short, abrupt sounds that could be viewed as disturbances, such as noise events created by infrequent and loud military jet overflights that alter the behavior and time budgets of harlequin ducks (Histrionicus histrionicus; WebFigure I; Goudie 2006). For disturbance sounds, exposure metrics that capture each sound event's maximum power $\left(\mathrm{L}_{\max }\right.$; WebFigure Ia) and the rate at which power rises from the lowest detectable level to its maximum are important (ie onset; Figure 2). $L_{\max }$ values are often reported without stating the frequency weighting; in these cases, A-weighting (a human-centric curve) is assumed, which may be inappropriate for many animals.

In contrast, quantification of chronic noise can best be served with time-averaged values such as $L_{e q}($ WebFigure $I b)$. $L_{e q}$ is typically calculated over 24 hours; however, many studies fail to report over what time period $L_{\text {eq }}$ values were integrated and a 24-hr integration is assumed, which may not be appropriate for many ecological questions. For example, for a species that is sensitive to traffic noise, such as the white-breasted nuthatch (Sitta carolinensis; WebFigure Ib; Goodwin and Shriver 20I I), it may be best to truncate the time interval to the hours of biological interest, such as during dawn chorus. Limiting frequency analyses to the hearing or vocal range of the target species or community may also be beneficial (eg Halfwerk et al. 20I lb). Future studies should aim to use biologically relevant integration times and report these details.

Best practices will include simultaneous acquisition of high-quality audio recordings along with multiple sound level measurements to offer unconstrained opportunities to investigate alternative spectral filtering, time integration, and additional measurements, such as order statistics indicating the percentage of time above a certain decibel level or metrics reflective of the sound event's predictability (Figure 2). Carefully considering how these temporal, intensity, and frequency features (Figure $2 b$ ) interact will help investigators identify where along the disturbance-interference continuum (Figure 2a) the stimulus is most likely to fall and will help identify the most likely behavioral responses (Figure 3 ).

Above all, to maximize interpretability of results, facilitate comparisons among studies, and provide meaningful data for conservation measures, it is critical to explicitly report the acoustic metrics used in each study to describe species responses. Additional sound metric and terminology details can be found in Barber et al. (20I I) and Pater et al. (2009). 


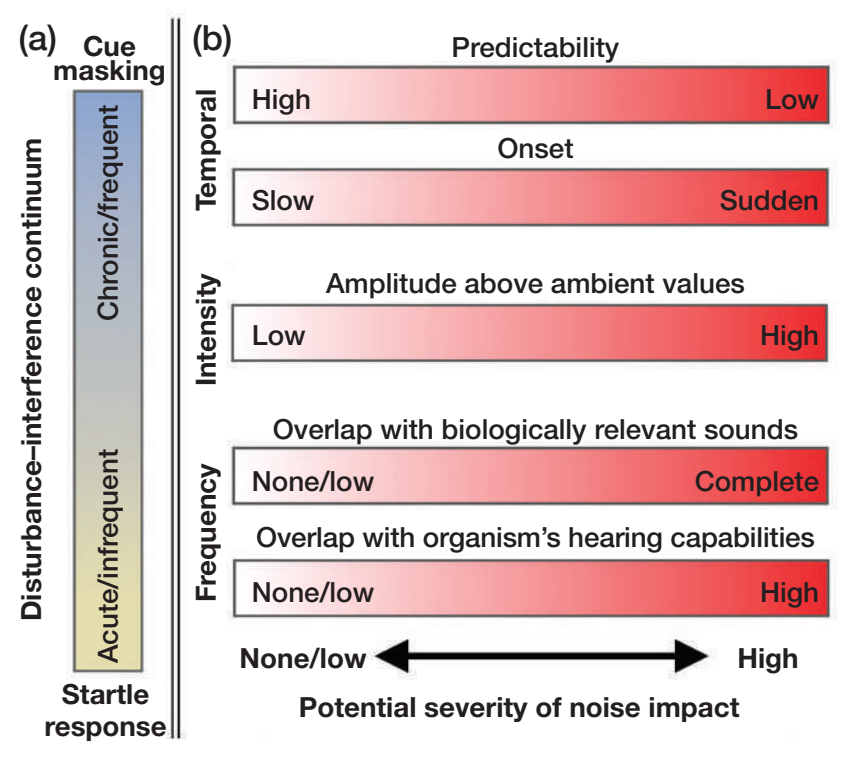

Figure 2. (a) The disturbance-interference continuum can range from acute or infrequent noise stimuli that will likely trigger startle or hide responses to frequent or chronic noises that interfere with cue detection. (b) The severity of an impact from a noise stimulus will depend on the temporal, intensity, and frequency features of the stimulus.

important behavioral adaptation that permits this species to overcome unfavorable acoustic conditions, the consequences of shifting the timing of song delivery are unknown. The effects of signal timing on mate attraction or territorial defense may be just as important to fitness as other signal features (eg frequency, syntax). Changes in the timing of song delivery of less than one hour can break down signaler-receiver coordination so that conspecific males do not recognize species-specific signals (Luther 2008). If signaler-receiver coordination is disrupted between singing males and responsive females, the behavioral flexibility that permits shifts in signal timing in response to noise may possibly be maladaptive.

Sleep is an important factor and follows a strong temporal profile. Although a substantial body of research has investigated the impact of noise on sleep in humans, scant information is available regarding its effects in other animals (reviewed in Kight and Swaddle 2011). Understanding the importance of sleep disruption on overall fitness is critical as we might expect detrimental influences even for species not typically described as dependent upon hearing (eg visually oriented predators such as raptors).

\section{Alterations in spatial distributions or movements}

Among the most obvious responses to noise are site abandonment and decreases in spatial abundance. These metrics may also be easiest and least costly to quantify, which perhaps explains why there are many such examples in the literature (eg Bayne et al. 2008; Eigenbrod et al. 2008; Francis et al. 2009). However, noise itself can affect an investigator's ability to measure responses to noise. For example, increases in continuous noise of 5-10 decibels ( $\mathrm{dB}, \mathrm{A}$-weighted; Panel 1) above baseline can reduce bird numbers during standard bird surveys by one-half, greatly biasing measures of site occupancy and abundance (Ortega and Francis 2012). If not carefully considered, this detection problem could bias subsequent interpretations and management efforts.

Despite the known effects of noise on population sizes, there is still considerable evidence to suggest that animals may abandon areas when frequent or chronic noise stimuli interfere with cue detection or when more variable sounds are perceived as threats (Bayne et al. 2008; Goodwin and Shriver 2011; Blickley et al. 2012a). Birds with low-frequency vocalizations experience more acoustic interference from chronic low-frequency anthropogenic noise and therefore exhibit stronger negative responses to noise in their habitat use than birds with high-frequency vocalizations that experience less acoustic interference (Figure 3e; Francis et al. 2011a). These masking effects can be spatially extensive, potentially impairing communication at distances ranging from 0.5 to $1.0 \mathrm{~km}$ or farther from the noise source (Blickley and Patricelli 2012). Furthermore, changes in spatial distributions due to noise's effect on cue detection are not restricted to intraspecific communication; for instance, greater mouse-eared bats (Myotis myotis), which locate terrestrial prey based on sounds they generate when walking, also avoid hunting in noisy areas (Figure 3f; Schaub et al. 2008). In addition to disrupting cue detection at the intra- and interspecific level, ambient noise may also interfere with cue detection used for movement at larger spatial scales. Some frog species use conspecific calls to locate appropriate breeding habitat, while some newt species use heterospecific calls for the same purpose (reviewed in Slabbekoorn and Bouton 2008). Whether noise exposure impedes animals from using such acoustic beacons to locate critical resources (eg water, food, habitat) is unknown and should be a focus of future research.

Site abandonment or decreases in population numbers can also occur in response to unpredictable, erratic, or sudden sounds, which are perceived as threats (Figure 3d). For example, greater sage grouse (Centrocercus urophasianus) lek attendance declines at a higher rate in response to experimentally introduced intermittent road noise than to continuous noise (Blickley et al. 2012a), suggesting that sage grouse site occupancy may depend more on perceived risk than on masking of acoustic cues. Nevertheless, masking of communication may have other consequences (Figure 1).

Species undoubtedly differ in their sensitivities to disruptive sounds, but individuals within a population also show such differences (Bejder et al. 2006). Individuals can 


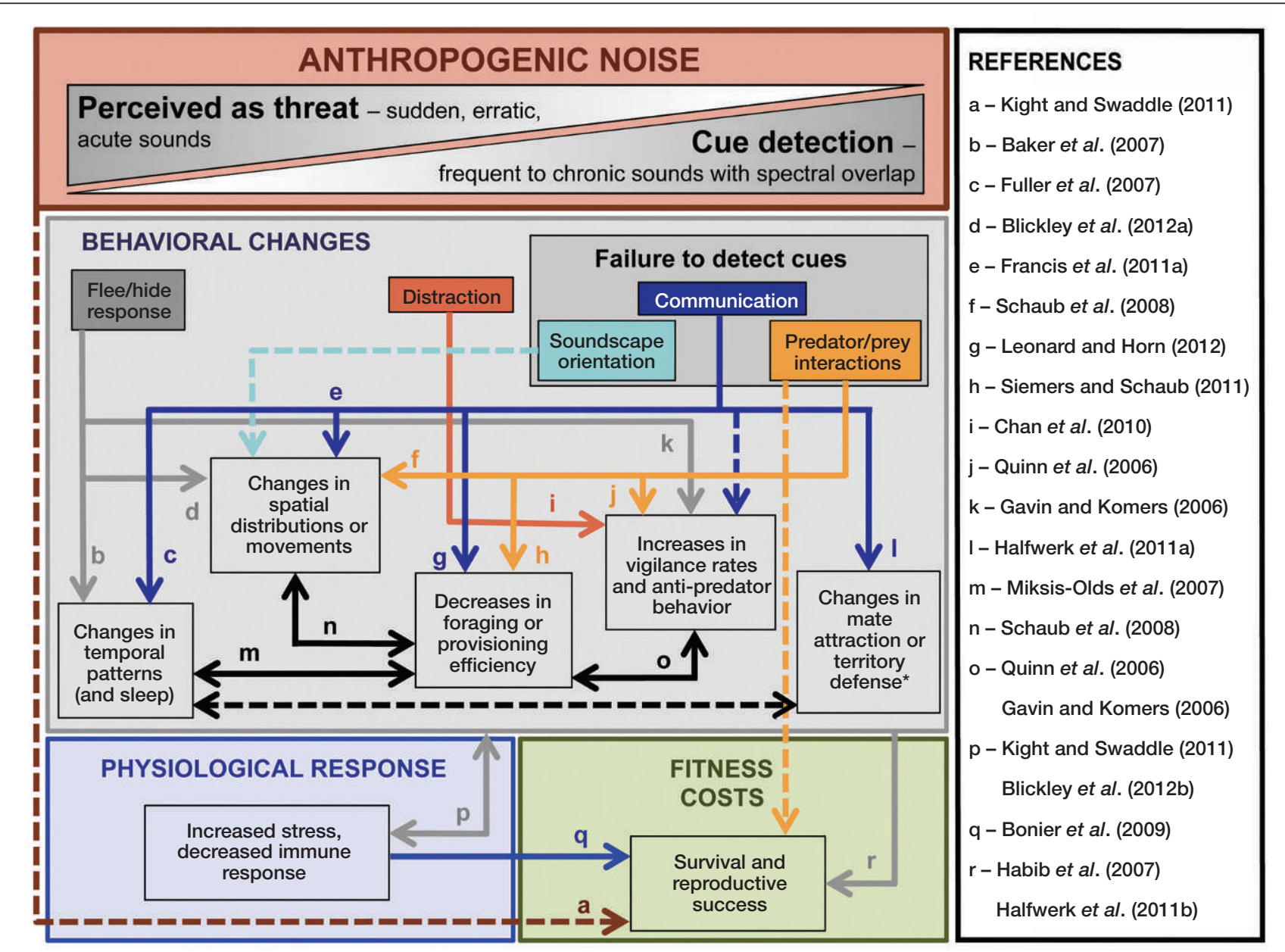

Figure 3. Conceptual framework for understanding how noise stimuli-perceived as a threat or interfering with cue detection (the disturbance-interference continuum) - can elicit behavioral responses that have direct consequences for fitness or via a physiological stress response, which can also feed back to behavioral changes. Startle/hide responses are more likely to occur in response to noise stimuli that are perceived as a threat (acute, erratic, or sudden sounds). Problems arising from a failure to detect cues are more likely to occur when noise stimuli are chronic and overlap with biologically relevant cues used for communication, orientation, and predator/prey detection. Problems arising from distraction may occur as a result of sounds with features ranging from those that interfere with cue detection to those that are perceived as threats. Lowercase letters indicate studies (listed on the right) providing evidence for the link made for each arrow. Dashed arrows signify a link that we predict as important but for which no current evidence exists. The asterisk denotes that which could result from a change in behavior or a failure to change behavior in response to noise.

vary greatly in their behavioral responses to stimuli, which may explain the variations in their ability to cope with environmental change (Sih et al. 2004). The redistribution of sensitive and tolerant individuals across the landscape may not appear to be a problem. However, in the case of social animals, where group living provides protection from predation, the loss of sensitive individuals from the group through site abandonment could increase predation risk for the group as a whole through the removal of the most vigilant group members. These sensitive individuals, who are now isolated from the group, lose the benefit of safety in numbers. Depending on population structure and the scale at which these individuals are displaced by noise, genetic diversity may be reduced because traits that govern risk-averse (shy/sensi- tive) and risk-prone (bold) behaviors can be heritable (Dingemanse et al. 2002).

Site abandonment and changes in abundance provide only a limited understanding of how noise can impact wildlife populations and communities. Importantly, abundance can also be misleading because areas where individuals are abundant do not always translate into high fitness for those individuals (eg Johnson and Temple 1986). Using such evidence to conclude that noise has no impact is problematic; individuals may not have alternative areas to occupy or other responses (survival, mating success, reproductive output) may be negatively affected by noise even when abundance is high (Figure 1a). These possibilities are especially likely when a noise stimulus is new and demographic processes 
have not had time to impact population size or when the population in an area that is exposed to noise is supplemented by individuals from elsewhere (ie sourcesink dynamics).

\section{Decreases in foraging or provisioning efficiency and increased vigilance and anti-predator behavior}

Noise can impair foraging and provisioning rates directly (Figure 3, g and h) or indirectly as a consequence of increased vigilance and anti-predator behavior (Figure 3, $\mathrm{i}-\mathrm{k}, \mathrm{o})$. When noise is perceived as a threat, an organism may miss foraging opportunities ("missed opportunity cost"; Brown 1999) while hiding or as a result of maintaining increased vigilance (Figure 3k; Gavin and Komers 2006). Missed opportunities can also occur when noise interferes with cue detection. For instance, nestling tree swallows (Tachycineta bicolor) exposed to noise beg less in response to recorded playbacks of parents arriving at nests (eg calls, movement, sounds) than nestlings in quiet conditions, presumably because the ambient noise masks parent-arrival sounds (Figure 3g; Leonard and Horn 2012). Unfortunately, this study did not determine whether missed provisioning opportunities translated into costs, such as reduced nestling mass or fledging success.

Noise that interferes with cue detection can also hamper predators' hunting abilities. For example, among greater mouse-eared bats, search time for prey was shown to increase and hunting success to decrease with exposure to experimental traffic noise (Figure 3h; Siemers and Schaub 2011). This decrease in foraging success may explain why some predators avoid noisy areas (Figure 3n; eg Schaub et al. 2008; Francis et al. 2009). Noise also impairs foraging in three-spined sticklebacks (Gasterosteus aculeatus), resulting in more unsuccessful hunting attempts (Purser and Radford 2011). Noise also possibly interferes with the ability of prey species to hear approaching predators, which could impact fitness directly. Although likely, elevated predation risk due to noise has yet to be demonstrated, but some evidence does suggest that animals exposed to noise behave as though they are at greater risk of predation. For example, in the chaffinch (Fringilla coelebs), continuous noise impairs auditory surveillance, triggering increased visual surveillance, as a result of which the birds spend less time foraging (Figure 3j; Quinn et al. 2006). Noise that serves as a distraction may also lead to an increased latency in predator-escape response (Figure 3i; Chan et al. 2010), potentially compromising survival. Both distraction and elevated vigilance could also cause a decrease in foraging rates and success (ie a trade-off; Figure 3o; Gavin and Komers 2006; Quinn et al. 2006). Collectively, these studies suggest that both interference noise and noise perceived as a threat decrease the rate and frequency at which organisms obtain food. Studies aimed at understanding the extent to which these behavioral shifts represent a metabolic expense (relevant to survival and reproductive success) will help to reveal the hidden costs of noise exposure.

\section{Changes in mate attraction and territorial defense}

The most direct way in which noise may alter an individual's ability to attract mates or defend its territory is through energetic masking, in which potential receivers are simply unable to hear another individual's acoustic signals through noise that is frequent or continuous during important temporal signaling windows. Changes made to acoustic signals appear to be an adaptive behavioral adjustment that permits individuals to communicate under noisy conditions (eg Fuller et al. 2007; Gross et al. 2010; Francis et al. 2011b), yet these shifts could also incur a cost. In noisy areas, female great tits (Parus major) more readily detect male songs sung at higher frequencies than females typically prefer (Halfwerk et al. 2011a). However, males who sing predominately at higher frequencies experience higher rates of cuckoldry (Figure 31). Great tits breeding in noisy areas also have smaller clutches and fewer fledglings (Halfwerk et al. 2011b); similarly, eastern bluebirds (Sialia sialis) experience decreased productivity when nesting in areas with elevated noise levels (Kight et al. 2012). Paired with patterns of decreased pairing success in noisy areas (Habib et al. 2007; Gross et al. 2010), these studies suggest that short-term signal adjustments in response to anthropogenic noise might function as evolutionary traps (eg Schlaepfer et al. 2002) in which behavioral responses to novel acoustic stimuli could be maladaptive. That is, behavioral shifts to be heard in noisy areas may come with the cost of compromising the attractiveness of the signal to potential mates. This possibility remains to be tested against other potential explanations for declines in pairing or reproductive success, but emphasizes why investigators should measure aspects of fitness in noise-impact studies rather than simply documenting changes in site occupancy or abundance.

Finally, although the list of species known to shift their signals in response to noise is growing, there is at least one frog species and some bird species that do not alter their vocalizations in response to noise (eg $\mathrm{Hu}$ and Cardoso 2010; Love and Bee 2010; Francis et al. 2011b). More work is needed to provide a thorough understanding of the phylogenetic distribution of noise-dependent vocal change and researchers should strive to publish negative results, as knowledge of the apparent absence of these behavioral modifications is just as important as knowledge of their presence.

\section{Linking behavioral changes, physiological responses, and fitness costs}

The behavioral changes mentioned above can have direct consequences for fitness (Figure 3r), such as reduced pairing success (Habib et al. 2007) or reduced 
reproductive success (Halfwerk et al. 2011b). However, behavior can influence, and be influenced by, physiological responses (Figure 3p; Kight and Swaddle 2011), which in turn can affect fitness (Figure 3q; Bonier et al. 2009). Kight and Swaddle (2011) reviewed many links between noise, physiological stress, and behavioral change, so we only briefly mention them here.

It is well known that increased physiological stress affects fitness (Figure 3q); yet, to our knowledge, a direct link between increased physiological stress due to noise and decreased survival or reproductive success has not been shown in wild animals. The best evidence for this potential link comes from two studies. In one, Blickley et al. (2012b) found that greater sage grouse on leks exposed to experimental playback of continuous natural gas drilling noise or intermittent road noise had higher fecal glucocorticoid metabolites (fGMs) than individuals on control leks. The authors suggested that masking of cues likely resulted in elevated stress levels, inhibiting social interactions or leading to a heightened perception of predation risk. In the other, Hayward et al. (2011) showed that experimental exposure to motorcycle traffic and motorcycle noise increased fGMs in northern spotted owls (Strix occidentalis caurina). In an observational component of the same study, spotted owls nesting in areas with higher levels of traffic noise fledged fewer offspring, even though they did not have elevated fGMs, suggesting that the effects of road noise may have been offset by greater prey availability in noisy areas. These two studies demonstrate that noise may lead to decreased fitness in sage grouse and spotted owls, and also clearly indicate that more research is needed to determine how noise exposure, physiological stress, and fitness are linked in wild populations.

\section{Scaling up behavioral responses}

Here, we have focused on effects of noise exposure at the level of the individual; however, studies that integrate individual behavior, population responses among multiple species, and species interactions are critical to understanding the cumulative, community-level consequences of noise. Measures of species richness are a good starting point, but may be misleading because species may respond negatively, positively, or not at all to sound stimuli (Bayne et al. 2008; Francis et al. 2009), individuals within a single species may respond differently to the same stimulus (Sih et al. 2004), and individuals that remain in noisy areas may suffer from one or more of the fitness costs discussed above. This variation within and among species in response to noise guarantees that communities in noisy areas will not always be subsets of the species that make up communities in comparable quiet areas. Researchers should couple standard measures of richness and alpha (local) diversity with beta-diversity metrics that reflect variations in the composition of species within communities and among sites.
Nevertheless, additional investigations will be needed to understand why species respond to sound stimuli as they do. Settlement patterns may not hinge on the intensity of noise, but are perhaps due to the presence or absence of cues indicating the presence of predators and heterospecific competitors (Francis et al. 2009). These other species (ie predators or competitors) may have unique settlement patterns in response to noise and will complicate efforts to measure how noise directly affects the species of interest. Disentangling these interactions will also be essential to understanding the consequences of noise exposure for organisms that are not directly impacted by noise, such as plants that depend on noise-sensitive faunal taxa (Francis et al. 2012) or animals whose hearing range is not tuned to a particular frequency that makes up a sound stimulus.

\section{- Conclusions}

Both policy and scientific literature have often oversimplified the effects of noise on wild animals, typically suggesting that species either are sensitive and abandon noisy areas or are not and remain. In our experience with stakeholders, habituation is an oft-cited reason for persistence and an absence of noise impacts, yet research on other stressors indicates that acclimation to a stressor might not release an organism from costs to fitness (Romero et al. 2009). Additionally, we have shown how behavioral modifications among individuals confronted with noise - even those individuals that outwardly appear to habituate - can lead to decreased fitness. Challenging the assumption that habituation to noise equals "no impact" will be difficult, but it will also be a critical component in revealing how a range of behavioral mechanisms link noise exposure to fitness costs. Ideally, we need to predict which combination of noise characteristics and behavioral contexts are most detrimental and under what circumstances behavioral changes affect fitness directly or indirectly. This will require an array of experimental and observational approaches and frameworks that complement the conceptual structure presented here (Figure 3). Other promising frameworks include the risk-disturbance hypothesis (Frid and Dill 2002), which provides an avenue for understanding energetic costs associated with wildlife responses to noise disturbances that are perceived as threats. Studies evaluating aspects of habitat selection and acoustic communication in response to noise may find it useful to frame questions in terms of ecological and evolutionary traps (Schlaepfer et al. 2002). Furthermore, investigators should strive to measure responses along a range of noise exposure levels to reveal the shape of response curves (eg threshold, linear) because these details will be indispensable to resource managers and policy makers when establishing and modifying regulatory limits that reflect the ecological effects of noise exposure.

An increase in anthropogenic noise levels is only one of many threats to biodiversity on which ecologists and policy makers should focus their attention. However, rel- 
ative to other conservation problems, noise may also offer readily available solutions, which, if implemented, could lead to major, measurable improvements for both wildlife and people. For example, use of noise-attenuating walls could reduce the area of a landscape exposed to elevated noise levels from natural gas extraction activities by as much as 70\% (Francis et al. 2011c) and similar solutions exist for mitigating noise from roadways and cities (Code of Federal Regulations 2010). These mitigation efforts could come with drawbacks; for instance, noise-attenuating walls near roads could restrict the movement of wildlife and impede gene flow. Nevertheless, as we develop a better understanding of the ecological effects of noise, implementation of mitigation efforts can begin in many well-studied and high-priority systems (eg oil and gas developments in natural areas, transportation networks in national parks), where benefits outweigh the potential costs. In addition to protecting contiguous natural habitat, reducing noise exposure in and around developed areas will not only benefit wildlife populations and diversity, but will also provide adjacent human populations with the suite of physiological benefits afforded by living in a quieter community.

\section{Acknowledgements}

We thank J Bunkley, K Fristrup, A Keener, N Kleist, B Leavell, T Mason, C McClure, and $\mathrm{H}$ Ware for comments on earlier versions of this manuscript. CDF thanks the National Evolutionary Synthesis Center (NSF EF0905606) for support and JRB acknowledges support from the Natural Sounds and Night Skies Division of the National Park Service (CESU H8R07060001).

\section{References}

Baker PJ, Dowding CV, Molony SE, et al. 2007. Activity patterns of urban red foxes (Vulpes vulpes) reduce the risk of trafficinduced mortality. Behav Ecol 18: 716-24.

Barber JR, Crooks KR, and Fristrup KM. 2010. The costs of chronic noise exposure for terrestrial organisms. Trends Ecol Evol 25: 180-89.

Barber J, Burdett C, Reed S, et al. 2011. Anthropogenic noise exposure in protected natural areas: estimating the scale of ecological consequences. Landscape Ecol 26: 1281-95.

Bayne EM, Habib L, and Boutin S. 2008. Impacts of chronic anthropogenic noise from energy-sector activity on abundance of songbirds in the boreal forest. Conserv Biol 22: 1186-93.

Bejder L, Samuels A, Whitehead H, and Gales N. 2006. Interpreting short-term behavioural responses to disturbance within a longitudinal perspective. Anim Behav 72: 1149-58.

Blickley JL and Patricelli GL. 2012. Potential acoustic masking of greater sage-grouse display components by chronic industrial noise. Ornithol Monogr 74: 23-35.

Blickley JL, Blackwood D, and Patricelli GL. 2012a. Experimental evidence for the effects of chronic anthropogenic noise on abundance of greater sage-grouse at leks. Conserv Biol 26: 461-71.

Blickley JL, Word KR, Krakauer AH, et al. 2012b. Experimental chronic noise is related to elevated fecal corticosteroid metabolites in lekking male greater sage-grouse (Centrocercus urophasianus). PLoS ONE 7: e50462.
Bonier F, Moore IT, Martin PR, and Robertson RJ. 2009. The relationship between fitness and baseline glucocorticoids in a passerine bird. Gen Comp Endocr 163: 208-13.

Brown JS. 1999. Vigilance, patch use and habitat selection: foraging under predation risk. Evol Ecol Res 1: 49-71.

Chan AAYH, Stahlman WD, Garlick D, et al. 2010. Increased amplitude and duration of acoustic stimuli enhance distraction. Anim Behav 80: 1075-79.

Code of Federal Regulations. 2010. Procedures for abatement of highway traffic noise and construction noise. Washington, DC: US Government Printing Office. 23 CFR \$772.17. www.ecfr. gov/cgi-bin/text-idx?c=ecfr\&SID $=$ cd313d7dd374014a $6041944112 \mathrm{~b} 4456 \mathrm{~b} \& \operatorname{rgn}=\operatorname{div} 5 \&$ view $=$ text $\&$ node $=23: 1.0 .1 .8$. 44\&idno=23\#23:1.0.1.8.44.0.1.9. Viewed on 29 Mar 2013.

Dingemanse NJ, Both C, Drent PJ, et al. 2002. Repeatability and heritability of exploratory behaviour in great tits from the wild. Anim Behav 64: 929-38.

Dooling RJ and Popper AN. 2007. The effects of highway noise on birds. Sacramento, CA: California Department of Transportation.

Eigenbrod F, Hecnar SJ, and Fahrig L. 2008. The relative effects of road traffic and forest cover on anuran populations. Biol Conserv 141: 35-46.

Ellison WT, Southall BL, Clark CW, and Frankel AS. 2012. A new context-based approach to assess marine mammal behavioral responses to anthropogenic sounds. Conserv Biol 26: 21-28.

Francis CD, Kleist NJ, Ortega CP, and Cruz A. 2012. Noise pollution alters ecological services: enhanced pollination and disrupted seed dispersal. P Roy Soc B-Biol Sci 279: 2727-35.

Francis CD, Ortega CP, and Cruz A. 2009. Noise pollution changes avian communities and species interactions. Curr Biol 19: $1415-19$

Francis CD, Ortega CP, and Cruz A. 2011a. Noise pollution filters bird communities based on vocal frequency. PLoS ONE 6: e27052.

Francis CD, Ortega CP, and Cruz A. 2011b. Vocal frequency change reflects different responses to anthropogenic noise in two suboscine tyrant flycatchers. P Roy Soc B-Biol Sci 278: 2025-31.

Francis CD, Paritsis J, Ortega CP, and Cruz A. 2011c. Landscape patterns of avian habitat use and nest success are affected by chronic gas well compressor noise. Landscape Ecol 26: 1269-80.

Frid A and Dill LM. 2002. Human-caused disturbance stimuli as a form of predation risk. Conserv Ecol 6: 11.

Fuller RA, Warren PH, and Gaston KJ. 2007. Daytime noise predicts nocturnal singing in urban robins. Biol Lett 3: 368-70.

Gavin SD and Komers PE. 2006. Do pronghorn (Antilocapra americana) perceive roads as a predation risk? Can J Zool 84: 1775-80.

Goodwin SH and Shriver WG. 2011. Effects of traffic noise on occupancy patterns of forest birds. Conserv Biol 25: 406-11.

Goudie RI. 2006. Multivariate behavioural response of harlequin ducks to aircraft disturbance in Labrador. Environ Conserv 33: 28-35.

Gross K, Pasinelli G, and Kunc HP. 2010. Behavioral plasticity allows short-term adjustment to a novel environment. Am Nat 176: 456-64.

Habib L, Bayne EM, and Boutin S. 2007. Chronic industrial noise affects pairing success and age structure of ovenbirds Seiurus aurocapilla. J Appl Ecol 44: 176-84.

Halfwerk W, Bot S, Buikx J, et al. 2011a. Low-frequency songs lose their potency in noisy urban conditions. P Natl Acad Sci USA 108: 14549-54.

Halfwerk W, Holleman LJM, Lessells CM, and Slabbekoorn H. 2011b. Negative impact of traffic noise on avian reproductive success. J Appl Ecol 48: 210-19.

Hayward LS, Bowles AE, Ha JC, and Wasser SK. 2011. Impacts of 
acute and long-term vehicle exposure on physiology and reproductive success of the northern spotted owl. Ecosphere 2: 65 .

Hu Y and Cardoso GC. 2010. Which birds adjust the frequency of vocalizations in urban noise? Anim Behav 79: 863-67.

Johnson RG and Temple SA. 1986. Assessing habitat quality for birds nesting in fragmented tallgrass prairies. In: Verner J, Morrison ML, and Ralph CJ (Eds). Wildlife 2000: modeling habitat relationships of terrestrial vertebrates. Madison, WI: University of Wisconsin Press.

Kight CR and Swaddle JP. 2011. How and why environmental noise impacts animals: an integrative, mechanistic review. Ecol Lett 14: 1052-61.

Kight CR, Saha MS, and Swaddle JP. 2012. Anthropogenic noise is associated with reductions in the productivity of breeding eastern bluebirds (Sialia sialis). Ecol Appl 22: 1989-96.

Leonard ML and Horn AG. 2012. Ambient noise increases missed detections in nestling birds. Biol Lett 8: 530-32.

Love EK and Bee MA. 2010. An experimental test of noise-dependent voice amplitude regulation in Cope's grey treefrog, Hyla chrysoscelis. Anim Behav 80: 509-15.

Luther DA. 2008. Signaller: receiver coordination and the timing of communication in Amazonian birds. Biol Lett 4: 651-54.

Miksis-Olds JL, Donaghay PL, Miller JH, et al. 2007. Noise level correlates with manatee use of foraging habitats. J Acoust Soc Am 121: 3011-20.

Ortega CP and Francis CD. 2012. Effects of gas well compressor noise on ability to detect birds during surveys in northwest New Mexico. Ornithol Monogr 74: 78-90.

Pater LL, Grubb TG, and Delaney DK. 2009. Recommendations for improved assessment of noise impacts on wildlife. J Wildlife Manage 73: 788-95.

Patricelli GL and Blickley JL. 2006. Avian communication in urban noise: causes and consequences of vocal adjustment. Auk 123: 639-49.

Purser J and Radford AN. 2011. Acoustic noise induces attention shifts and reduces foraging performance in three-spined sticklebacks (Gasterosteus aculeatus). PLoS ONE 6: e17478.

Quinn JL, Whittingham MJ, Butler SJ, and Cresswell W. 2006. Noise, predation risk compensation and vigilance in the chaffinch Fringilla coelebs. J Avian Biol 37: 601-08.

Romero LM, Dickens MJ, and Cyr NE. 2009. The reactive scope model - a new model integrating homeostasis, allostasis, and stress. Horm Behav 55: 375-89.

Schaub A, Ostwald J, and Siemers BM. 2008. Foraging bats avoid noise. J Exp Biol 211: 3174-80.

Schlaepfer MA, Runge MC, and Sherman PW. 2002. Ecological and evolutionary traps. Trends Ecol Evol 17: 474-80.

Siemers BM and Schaub A. 2011. Hunting at the highway: traffic noise reduces foraging efficiency in acoustic predators. $P$ Roy Soc B-Biol Sci 278: 1646-52.

Sih A, Bell A, and Johnson JC. 2004. Behavioral syndromes: an ecological and evolutionary overview. Trends Ecol Evol 19: 372-78.

Slabbekoorn H and Bouton N. 2008. Soundscape orientation: a new field in need of sound investigation. Anim Behav 76: E5-E8.

Slabbekoorn H, Bouton N, van Opzeeland I, et al. 2010. A noisy spring: the impact of globally rising underwater sound levels on fish. Trends Ecol Evol 25: 419-27.

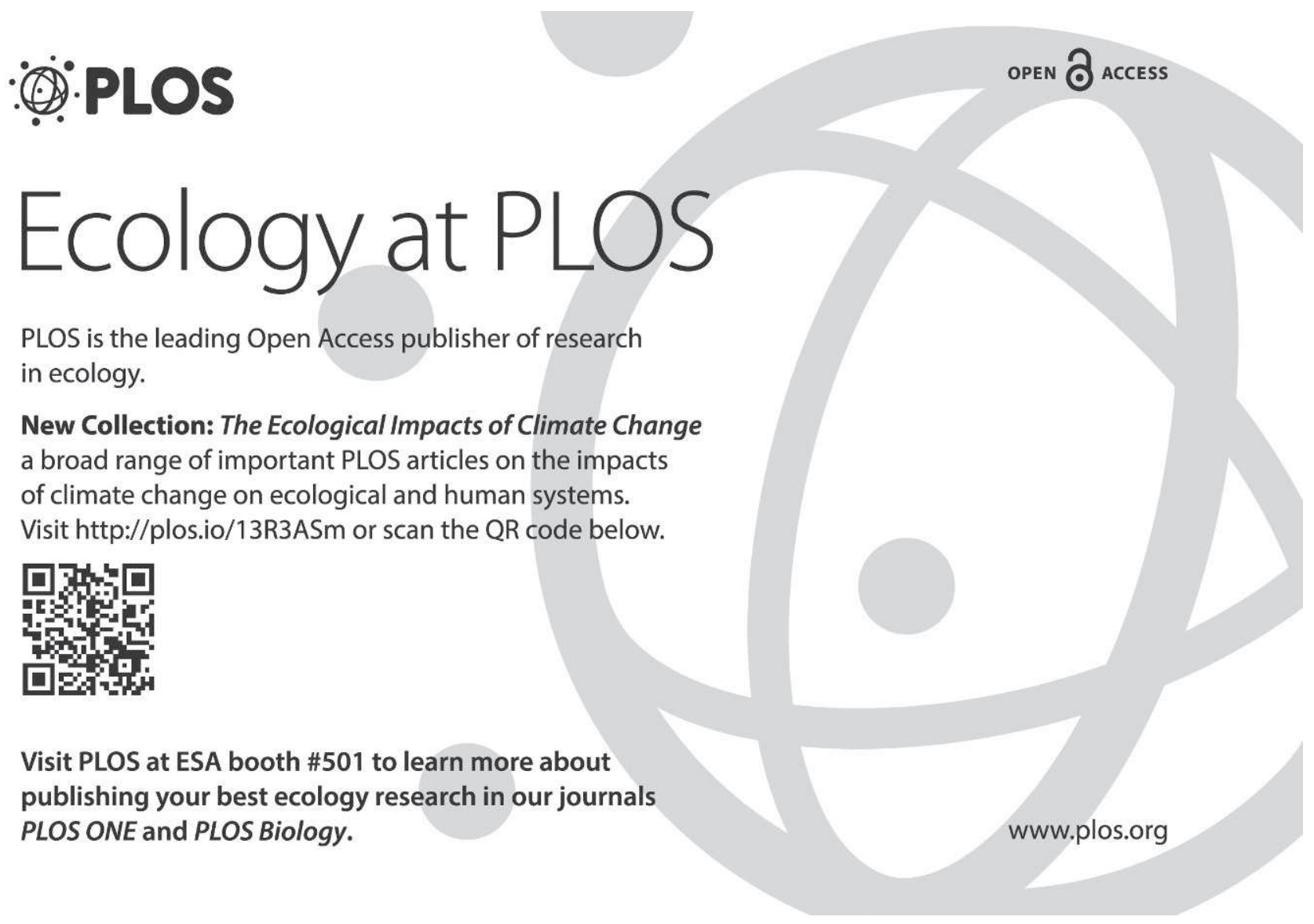

